Overview of equilibrium reconstruction on DIII-D using new measurements from an expanded motional Stark effect diagnostic

C.T. Holcomb, M.A. Makowski, S.L. Allen, W.H. Meyer, M.A. Van Zeeland

May 2, 2008

17th Topical Conference High-Temperature Plasma Diagnostics

Albuquerque, NM, United States

May 11, 2008 through May 15, 2008 
This document was prepared as an account of work sponsored by an agency of the United States government. Neither the United States government nor Lawrence Livermore National Security, LLC, nor any of their employees makes any warranty, expressed or implied, or assumes any legal liability or responsibility for the accuracy, completeness, or usefulness of any information, apparatus, product, or process disclosed, or represents that its use would not infringe privately owned rights. Reference herein to any specific commercial product, process, or service by trade name, trademark, manufacturer, or otherwise does not necessarily constitute or imply its endorsement, recommendation, or favoring by the United States government or Lawrence Livermore National Security, LLC. The views and opinions of authors expressed herein do not necessarily state or reflect those of the United States government or Lawrence Livermore National Security, LLC, and shall not be used for advertising or product endorsement purposes. 


\title{
Overview of equilibrium reconstruction on DIII-D using new measurements from an expanded motional Stark effect diagnostic
}

\author{
C.T. Holcomb, M.A. Makowski, S.L. Allen, W.H. Meyer \\ Lawrence Livermore National Laboratory, 7000 East Ave, Livermore, California 94550, \\ USA
}

M.A. Van Zeeland

General Atomics, PO Box 85608, San Diego, California 92186-5608, USA

(Received on

\begin{abstract}
Motional Stark effect (MSE) measurements constrain equilibrium reconstruction of DIII-D tokamak plasmas using the equilibrium code EFIT. In 2007, two new MSE arrays were brought online, bringing the system to three core arrays, two edge arrays, and 64 total channels. We present the first EFIT reconstructions using this expanded system. Safety factor and $E_{\mathrm{R}}$ profiles produced by fitting to data from the two new arrays and one of the other three agree well with independent measurements. Comparison of the data from the three arrays that view the core shows that one of the older arrays is inconsistent with the other two unless the measured calibration factors for this array are adjusted. The required adjustments depend on toroidal field and plasma current direction, and on still other uncertain factors that change as the plasma evolves. We discuss possible sources of calibration error for this array.
\end{abstract}

PACS: 52.55.Fa, 52.70.-m, 52.70.Kz 


\section{INTRODUCTION}

The DIII-D tokamak [1] now has five operating motional Stark effect (MSE) polarimeters [2] that provide the primary internal constraints for equilibrium reconstruction. The last two arrays built were brought online in 2007. These view a neutral beam injected counter to the plasma current. One array views the core, and the other the edge, and both use a single fold mirror. The design of these polarimeters and the expected measurement improvements are described in reference 2. In general, the geometry of the new views, henceforth labeled collectively as the "counter-MSE", is more favorable than the "radial-MSE" core view in terms of spatial resolution and $B_{\mathrm{Z}}$ and $E_{\mathrm{R}}$ uncertainty (Figs. 1, 2, and 3 in Ref. 2).

To test how well the counter-MSE meets these expectations we have produced EFIT [3] reconstructions for a wide variety of plasma discharge conditions using only the original tangential array ( $\phi=315^{\circ}$ in Ref. 2, Fig. 1) and the counter-MSE arrays ( $\phi=$ $195^{\circ}$ ). These would then be compared to reconstructions that used the tangential and radial $\left(\phi=15^{\circ}\right)$ arrays without the counter arrays. The remaining MSE array that views the edge $\left(\phi=45^{\circ}\right)$ is not considered here, as it has a coating on the plasma facing optic and typically gives unreliable results without a significant ad-hoc calibration adjustment. Replacement of this component is planned for later this year.

We find that using the tangential+counter MSE set of constraints in EFIT with a calibration without ad hoc adjustments produces current profiles that are in good agreement with independent measurements that infer current profile details - most notably MHD activity. This agreement holds for a wide variety of plasma discharges and toroidal current and field polarities. (The plasma current and toroidal field directions are 
independently reversible in DIII-D.) In contrast, the tangential+radial MSE set of constraints in EFIT does not produce good agreement with observed MHD without applying significant adjustments to the measured calibration. The adjusted calibrations must be arrived at empirically, and separate calibrations are needed for each field and current polarity. The new counter-MSE data thus identifies the radial array as the most likely source of systematic calibration error or measurement interpretation error.

In the next section, we discuss the calibration procedures that have been used on the DIII-D MSE diagnostic. This is followed by examples of EFIT reconstructions that use the counter-MSE with a calibration without ad hoc adjustments. These reconstructions are benchmarked by independent measurements. The last section shows the results of attempting to correct the radial array calibration using a series of benchmarked EFITs that rely on the tangential+counter array constraints and discusses possible sources of error affecting this array.

\section{CALIBRATION}

The DIII-D MSE polarimeters are calibrated using a four-parameter fitting function [4] in-situ. The formula to interpret the measurements is $S 1 / S 2=$ Gain $\bullet \tan \left[2\left(\gamma+\right.\right.$ Phase $+B_{\mathrm{T}}$ scale $\left.\left.\bullet B_{\mathrm{T}}\right)\right]+\mathrm{dc}$ offset, where $S 1$ and $S 2$ are the measured amplitudes of the second harmonics of the photo-elastic modulators, $B_{\mathrm{T}}$ is the vacuum toroidal magnetic field, and $\gamma$ is the angle of the electric field vector projected into the MSE optics (i.e. the quantity to be inferred). All fitting parameters except $B_{\mathrm{T}}$ scale are determined by placing a linear polarizer at the neutral beam-sightline intersection, and scanning through $360^{\circ} B_{\mathrm{T}}$ scale accounts for Faraday rotation, and it is determined by placing a stationary linear polarizer in the sightline at several values of 
$B_{\mathrm{T}}$. Calibrations determined from firing the neutral beam into a gas with vacuum magnetic fields applied have not been adequate, and recent atomic physics calculations confirm that the polarization state of the spectrum is different in this case [5].

When only the tangential, radial and edge arrays were available, this set of constraints produced EFITs that were inconsistent with observed MHD, or in some cases would not converge, unless the Phase of one or more arrays were empirically varied from the values determined in-situ. Typically all arrays were adjusted by an average of $0.3^{\circ}$, and separate final calibrations were required for each field and current polarity.

\section{EFITS USING COUNTER MSE}

The following example EFITs use only the tangential and counter-MSE channels as internal constraints. All cases use a calibration measured in-situ without any further empirical adjustments. Figure 1 shows data from a discharge with the standard $B_{\mathrm{T}}$ and $I_{\mathrm{p}}$ polarities. It compares the safety factor profile [Fig. 1(c)] ( $q$-profile) with a $41.1 \mathrm{kHz}$ rotating magnetic fluctuation observed on the pick-up coils [Fig. 1(a)]. Fourier analysis indicates the best-fit poloidal/toroidal mode numbers are $m / n=5 / 3$. According to MHD theory [6,7], the magnetic island producing this fluctuation should be centered about the $q=5 / 3$ flux surface, which is at $R=2.04 \mathrm{~m}$ in Fig. 1(c), and rotating toroidally at approximately $41.1 \mathrm{kHz} / n$. The charge exchange recombination (CER) diagnostic carbon impurity rotation profile [Fig. 1(b)] shows a $13.7 \mathrm{kHz}$ rotation $(41.1 \mathrm{kHz}$ divided by $n=3$ ) approximately at $2.02-2.04 \mathrm{~m}$. These observations form a consistent picture that locates the mode within 1-2 cm, which is approximately the spatial resolution of the MSE channels at this radius and the $65 \times 65$ EFIT grid spacing. 
It is possible to predict the value of $q_{\min }$ using electron cyclotron emission measurements of internal transport barriers that form when $q_{\min }$ is an integer and halfinteger [8]. Interferometer measurements also provide data on reverse shear Alfven eigenmode frequencies that evolve in a known way with $q_{\min }$ [9]. Figure 2(a) shows such measurements and the predicted $q_{\min }$ values at a number of times for a reverse- $B_{\mathrm{T}}$ discharge. Figure 2(b) shows the MSE constrained EFIT q-profiles at these same times. All these profiles agree well with the predictions from ECE and interferometry, except two which are at most 0.2 too low ( $t=530 \mathrm{~ms}$ and $t=1110 \mathrm{~ms}$ ).

Another check on the accuracy of the MSE calibration comes from including pressure profile constraints in the EFIT. If MSE measurements are a good constraint on the local toroidal current density $j_{\phi}=-R p^{\prime}-F F^{\prime} / \mu_{0} R$, then including additional pressure profile constraints may be expected to modify $p^{\prime}$ and $F F^{\prime}$ individually, but the resulting $j_{\phi}$ should not be grossly different. If it is - and this is sometimes the case when using an empirically adjusted MSE calibration - then either the MSE data is in error, the pressure data is in error, both are in error, or EFIT is somehow insufficient. Figure 3 shows profiles of the flux surface averaged parallel $j$ for a relatively extreme discharge with the plasma current reversed so up to five neutral beams are injected counter to it. This creates a very hollow current profile and broad pressure profile with internal transport barriers. The solid trace is the result of using edge magnetics and MSE constraints only, and the dashed trace uses these plus a total pressure constraint. The so-called kinetic EFIT confirms the gross features of the MSE-only EFIT in the core. The chief difference is in the edge where MSE channels have not yet been optimized and do not properly constrain the narrow bootstrap current peak at the H-mode pedestal. In this case, the kinetic EFIT 
has also not been set-up with enough spline knots in the edge $p^{\prime}$ or $F F^{\prime}$ to properly capture the bootstrap current here either.

Figure 4 compares the radial electric field determined from MSE and the same determined using CER for a DIII-D hybrid discharge. They agree well to within the MSE random error uncertainty of $\sim 10 \mathrm{kV} / \mathrm{m}$, except near the edge for the same reasons as the last example

\section{CALIBRATION OF THE RADIAL MSE ARRAY}

Figure 5 shows one dramatic example of how the radial array is inconsistent with the other core arrays when only an unadjusted calibration is applied. The points are the individual measured pitch angles $\gamma$ versus major radius in a standard monotonic $q$ profile, sawtoothing discharge. At the magnetic axis, $B_{z}=E_{R}=0$, so $\gamma=0$ from Eq. (1) of Ref. [1]. While the tangential and counter MSE arrays both interpolate $\gamma=0$ at about the same $R$, the radial array predicts $\gamma=0$ several centimeters away. Estimates of the error in $\gamma$ due to the spatial averaging of the radial array in a typical monotonic $q_{\min } \sim 1$ discharge are not enough to account for this misalignment [10].

This error would seem to be a simple systematic offset error in the calibration that could be eliminated by empirical adjustments to the Phase factors of the channels in this array. However a single set of adjustments that is universally applicable has not been found. If we take the preceding EFIT examples and others that fit to the tangential and counter MSE arrays as a set of benchmarked equilibriums, then we can calculate what Phase adjustment is required for each radial array channel to make it agree with an equilibrium. Figure 6 shows the resulting $\Delta \gamma$ 's versus their major radius position for nine benchmarked equilibriums. These represent all three polarities, $\left(+I_{\mathrm{p}},-B_{\mathrm{T}}\right),\left(+I_{\mathrm{p}},+B_{\mathrm{T}}\right)$, 
and $\left(-I_{\mathrm{p}},-B_{\mathrm{T}}\right)$, and a case at low, medium, and high $q_{\min }$ each. The source of the systematic error is clearly strongly dependent on field and current direction. $-I_{\mathrm{p}}$ also means mostly counter-injected neutral beams. The systematic error is also apparently weakly dependent on something in the plasma itself, as indicated by different corrections required within a specific $\left(I_{\mathrm{p}}, B_{\mathrm{T}}\right)$ polarity.

While the source of the systematic error afflicting the radial array is still unknown, these observations guide some speculation. Many possible calibration errors may be classified as geometric: perhaps the calibration polarized light source was not leveled correctly; perhaps the neutral beam geometry is not known accurately; spatial average effects; etc. All of these should produce a systematic error that is independent of the field and current direction. Faraday rotation in optics would depend on the field direction, but the radial array vacuum window is the same as, and in the same relative location as three other MSE array windows on systems that do not have these problems. A source of background polarized light might depend on plasma conditions. Reflections are one possibility. Another possibility is that emission from fast ions that undergo charge exchange recombination in the MSE viewing volume may, with the right viewing geometry, introduce background light with a net nonzero polarization fraction into the optics [11]. Such an effect has yet to be demonstrated on DIII-D, but it might explain the apparent dependence of the required calibration adjustment for this array on $q_{\min }$ and whether the neutral beams are mostly co or counter to $I_{\mathrm{p}}$ because the fast ion density is expected to depend on these factors [9]. 


\section{CONCLUSION}

We have shown that the use of two new MSE arrays with one of the older arrays as constraints in equilibrium reconstruction produces equilibria that are in good agreement with independent measurements and the expectations of MHD theory. These arrays are calibrated in-situ, and no further empirical adjustments to the calibration are required. This has made it possible to identify one of the older MSE arrays as being subject to systematic error that we are currently trying to understand, while at the same time improving our confidence in the equilibrium analysis in ongoing DIII-D experiments.

\section{Acknowledgment}

This work was performed under the auspices of the US Department of Energy by Lawrence Livermore National Laboratory under DE-AC52-07NA27344 and General Atomics under DE-FC02-04ER54698 . 


\section{References}

[1] J.L. Luxon, Nucl. Fusion 42, 614 (2002).

[2] C.T. Holcomb, M.A. Makowski, R.J. Jayakumar, S.A. Allen, R.M. Ellis, R. Geer, D. Behne, K.L. Morris, L.G. Seppala, and J.M. Moller, Rev. Sci. Instrum. 77, $10 \mathrm{E} 506$ (2006).

[3] L.L. Lao, J.R. Ferron, R.J. Groebner, W. Howl, H. St. John, E.J. Strait, and T.S. Taylor, Nucl. Fusion 30, 1035 (1990).

[4] M.A. Makowski, S.L. Allen, R. Ellis, R. Geer, R.J. Jayakumar, and J.M. Moller, Rev. Sci. Instrum. 76, 023706 (2005).

[5] M. Gu, C.T. Holcomb, R.J. Jayakumar, and S.L. Allen, J. Phys. B: At. Mol. Opt. Phys. 41, 095701 (2008)

[6] Z. Chang, J.D Callen, E.D. Fredrickson, R.V. Budny, C.C. Hegna et al., Phys. Rev. Lett. 74, 4663 (1995).

[7] R.J. La Haye, C.C. Petty, E.J. Strait, F.L. Waelbroeck, and H.R. Wilson, Phys. Plasmas 10, 3644 (2003).

[8] M.E. Austin, K.H. Burrell, R.E. Waltz, K.W. Gentle, P. Gohil, C.M. Greenfield, R.J. Groebner, W.W. Heidbrink, Y. Luo, J.E. Kinsey, M.A. Makowski, G.R. McKee, R. Nazikian, C.C. Petty, R. Prater, T.L. Rhodes, M.W. Shafer, and M.A. Van Zeeland, Phys. Plasmas 13, 082502 (2006).

[9] M.A. Van Zeeland, M.E. Austin, T.N. Carlstrom, T. Deterly, D.K. Finkenthal, C.T. Holcomb, R.J. Jayakumar, G.J. Kramer, M.A. Makowski, G.R. McKee, R. Nazikian, W.A. Peebles, T.L. Rhodes, W.M. Solomon, and E.J. Strait, Nucl. Fusion 46, S880S887 (2006). 
[10] M.A. Makowski, C.T. Holcomb, R.J. Jayakumar, and W. Meyer, Proc. of 16th Top. Conf. on High-Temperature Plasma Diagnostics, Williamsburg, Virginia, 2006, Paper THP42.

[11] S. Scott, J. Ko, I. Hutchinson, H. Yuh, Bull. Am. Phys. Soc. 52, 215 (2007). 


\section{List of Figure Captions}

Fig. 1. (a) $41 \mathrm{kHz} 5 / 3$ mode is located by CER rotation profile (b) at $R=2.04 \mathrm{~m}$, which agrees with (c) MSE $q$ profile.

Fig. 2. $q_{\min }$ evolution determined using ITB and RSAE observations by ECE and interferometry agree with MSE $q_{\text {min }}$.

Fig. 3. MSE-only EFIT and MSE plus kinetic EFIT current profiles are in good agreement.

Fig. 4. $E_{\mathrm{R}}$ profile determined by MSE and CER are in good agreement.

Fig. 5. Magnetic axis location predicted by tangential and counter MSE are in agreement, but radial MSE is not. Error bars indicate standard deviation of $10 \mathrm{~ms}$ averaging window (i.e. random uncertainty only).

Fig. 6. Required calibration adjustments for the radial MSE channels based on nine benchmarked EFITs. 

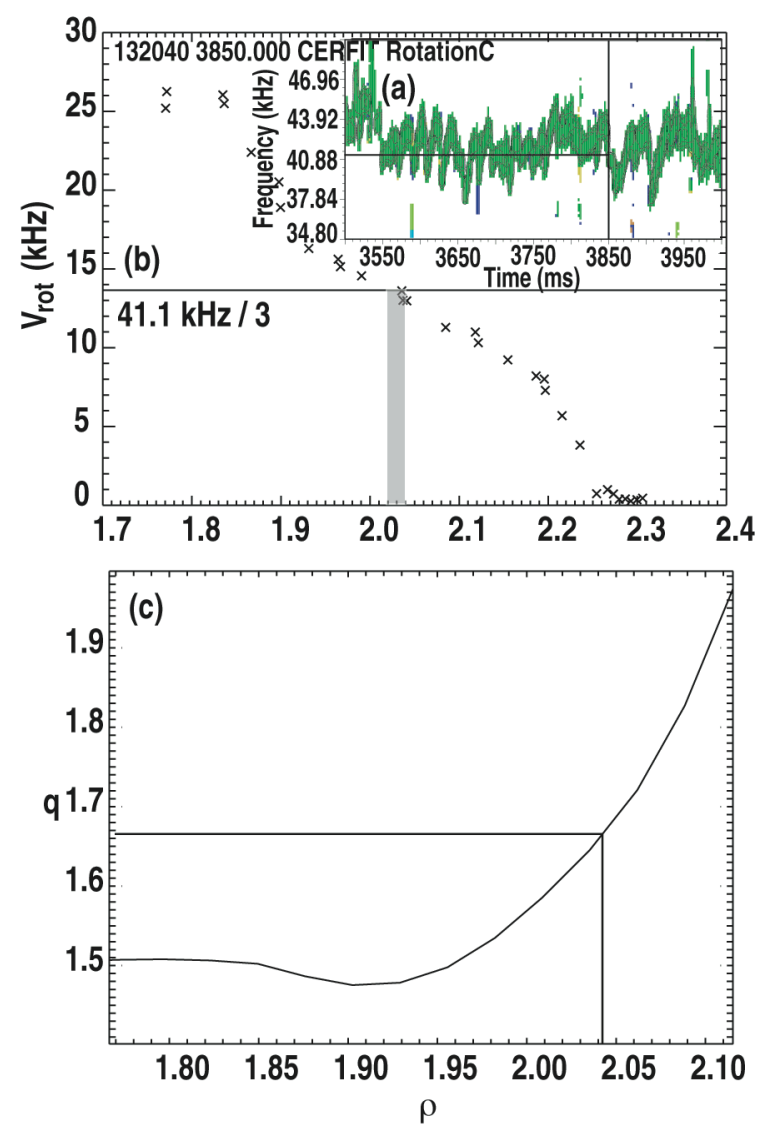

Fig. 1 

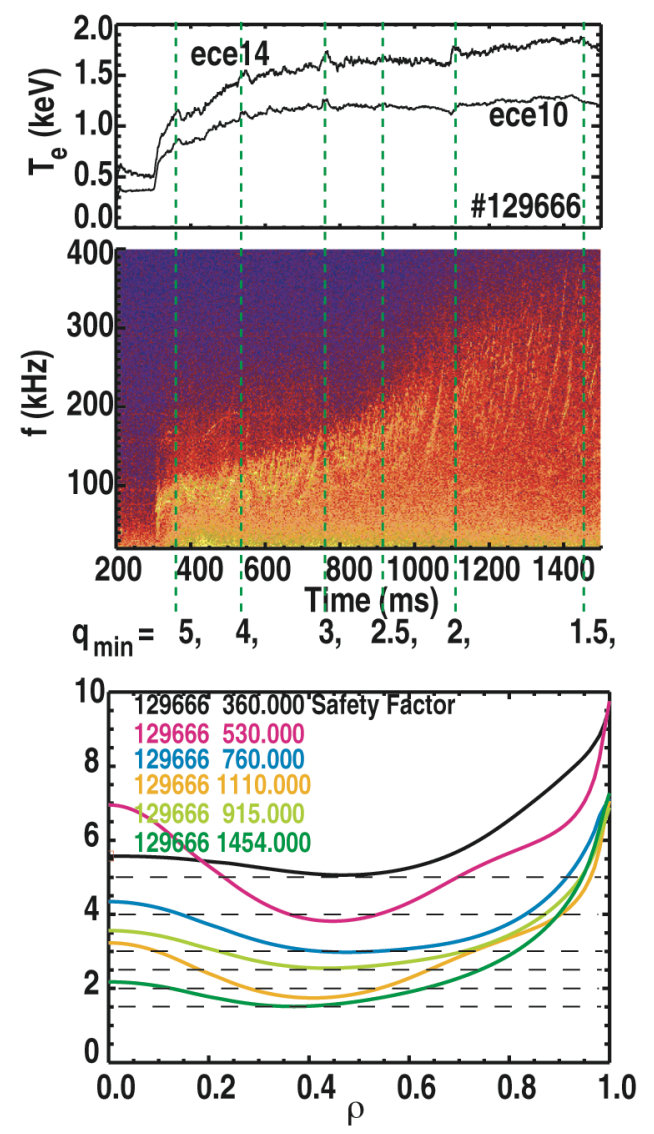

Fig. 2

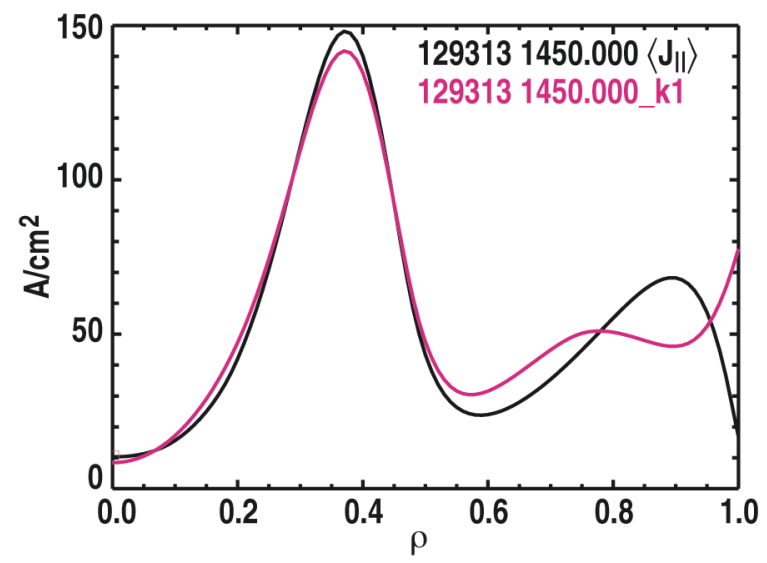

Fig. 3 


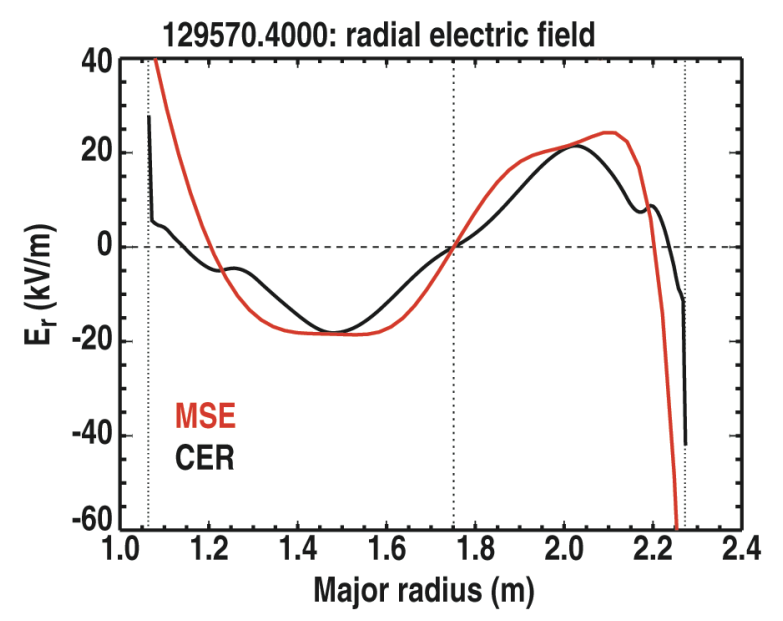

Fig. 4

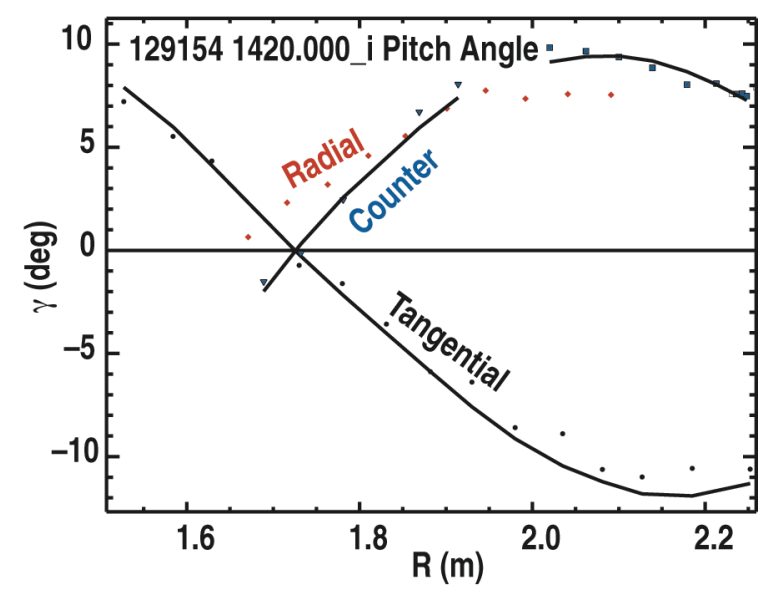

Fig. 5

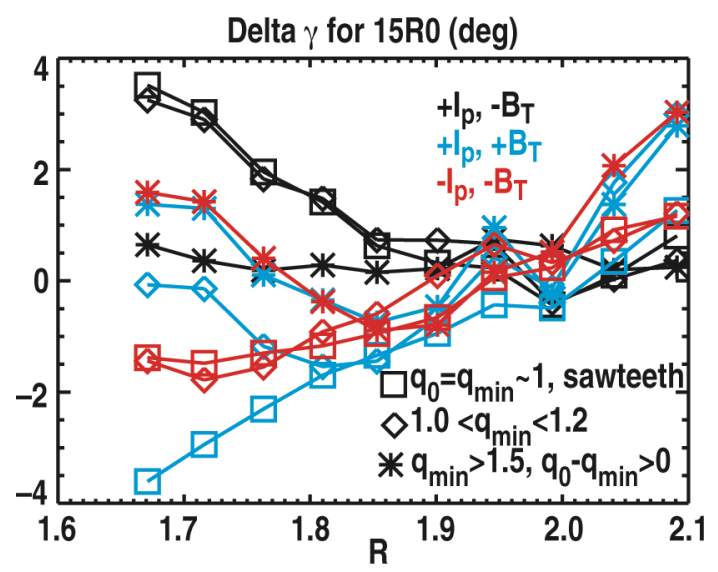

Fig. 6 